\title{
Aortic Surgery Symposium 2012 discussions-Panel 2 (sessions III and IV): Aortic arch
}

\author{
Moderator: Joseph E. Bavaria, MD, Philadelphia, Pa. \\ Panelists: Randall B. Griepp, MD, Mount Sinai School of Medicine, New York, NY; David Spielvogel, MD, \\ Westchester Medical Center, Valhalla, NY; Yukata Okita, MD, Kobe University, Kobe, Japan; Hitoshi Ogino, \\ $\mathrm{MD}, \mathrm{PhD}$, Department of Cardiovascular Surgery, National Cerebral and Cardiovascular Center, Suita, Japan; \\ Roberto Di Bartolomeo, MD, Universita di Bologna, Bologna, Italy; Martin Czerny, MD, University Hospital \\ Berne, Berne, Switzerland; Christian Hagl, MD, University of Munich, Munich, Germany; Yoshihiko Yokoi, \\ MD, Cardiovascular Surgery, Tokyo Women's Medical University, Tokyo, Japan; Cherrie Abraham, MD, Jewish \\ General Hospital \& McGill University, Montreal, Quebec, Canada; Joseph S. Coselli, MD, Texas Heart Institute, \\ Baylor College of Medicine, Houston, Tex; Jean E. Bachet, MD, Department of Cardiac Surgery, Zayed Military \\ Hospital, United Arab Emirates.
}

\section{Discussion}

Dr Bavaria. Cherrie, I will ask you a question first. You know that, especially in atherosclerotic aneurysms, the orifices of the innominate and the carotid are pretty loaded with plaque a lot of the time. Both the hybrid and the standard procedures avoid confronting that by inserting the branches distally. Chuter's original data showed a $25 \%$ stroke rate, or something equally horrible; that is why it was stopped. How are you going to deal with the special considerations regarding manipulations of the orifices of the aortic branches and stroke risk?

Dr Abraham. I agree; I am as concerned as you are. I think one of the problems with the Chuter device-and any procedure in which you are going retrograde with a large-profile delivery system-is that you are going to be rubbing against these atherosclerotic lesions. The advantage of the arch graft is the manipulation at that level is fairly minimal because you are only using catheters and wires, and then a fairly low profile stent-graft such as a Fluency or a Gore Viabahn.

I think, as I said, that severe atherosclerosis is a contraindication. If you see significant occlusive disease or calcification in the proximal trunks of these vessels, I don't think you should do this kind of graft. If the branch vessels are fairly clean or mildly atheromatous, however, I think you can get away with the branch technique. I am a little bit more worried about putting in fenestrated grafts, especially with the preloaded wire system, because they'll just rub across all that debris. But as Dr Yokoi showed, I am pretty impressed with the fact that maybe we don't need to use a preloaded wire or catheter to maintain alignment.

Dr D. Craig Miller (Stanford, Calif). All of us know that maternity is never in question, but paternity is suspect, and I shudder a little bit having had something to do 20 years ago with the advent of thoracic aortic stent-grafting. Whether I am the mother or the father, who knows, but I am responsible in some sense for what

Disclosures: Authors have nothing to disclose with regard to commercial support. Read at Aortic Surgery Symposium 2012, New York, NY, Thursday, April 26-27, 2012. Address for reprints: Eva B. Griepp, MD, Department of Cardiothoracic Surgery, Mount Sinai School of Medicine, New York, NY 10029 (E-mail: ebgriepp@aol.com). J Thorac Cardiovasc Surg 2013;145:S118-22 $0022-5223 / \$ 36.00$

Copyright (c) 2013 by The American Association for Thoracic Surgery http://dx.doi.org/10.1016/j.jtcvs.2012.11.081 we have seen today. And I have a very simple answer for you, Dr Abraham. You refer that patient to a surgeon who can do it right with reasonable results. I didn't see anything inoperable about that patient. And for those who are inoperable, there are more and more thoracic endovascular aortic repairs (TEVARs) being done, but fewer and fewer patients are living 5 years later because we are doing TEVARs in patients who are dying of other things. It is equivalent to cohort $\mathrm{C}$ for the percutaneous aortic valve, and it is futile therapy. So convince me that a patient is inoperable, and then maybe TEVAR is the thing to do. It is exciting, it is new, but the results I just don't think you can justify.

Now, let me ask my question of Joe and Martin. You both have the most wonderful platform to do it right if you can justify it, and I am not going to get into the debate that you can't do it the traditional way or Nick's way. If they are doing something in the ascending aorta and you are going to do a hybrid, I heard Martin say that you might come back another day and do a retrograde stent-graft. But why not do it antegrade at the same time, when you have so much better control of everything? Why would either of you do a 2-stage and come up from below with its risks, which you certainly don't have when you are in the ascending aorta anyway?

Dr Bavaria. I totally agree with antegrade versus retrograde stenting. After operating in the ascending aorta, we would do almost all of our cases with an antegrade stent. Occasionally, we will get into some issues that make it a little bit difficult technically; usually, if we do a retrograde stent, it is unplanned. We just get to the technical portion of the operation and we see that we can't put an antegrade stent in very easily, so we stop, and come back 2 or 3 or 4 days later and put in a retrograde stent. To be honest, there are some political issues with this operation in the hospital, and occasionally it is a primary vascular surgery patient and the surgeons like to put the stents in retrograde. But that is not really a medical reason.

Dr Czerny. Actually for us there are 2 reasons not to go for an antegrade delivery. The first is that you lose landing zone. If you insert the stent-graft through the ascending aorta with a side graft, you always lose at least $2 \mathrm{~cm}$ of what you have just gained by transposition.

And the second reason is that most prostheses are built and designed for retrograde delivery. There is actually just 1 prosthesis-the Gore prosthesis-for which I think from the 
design it doesn't matter whether you deploy it in an antegrade or retrograde fashion, because it opens up from the middle, and there are not bare springs on one side and not on the other. These are the 2 reasons for us to remain with the retrograde approach.

Dr Bavaria. I repeat, we usually do the antegrade approach.

Dr Miller. I don't buy those excuses. What is wrong with using the Gore antegrade?

Joe Coselli, you have been a proponent of going antegrade if you are there anyway. Do you see a reason to come back another day and buy those risks?

Dr Czerny. Regarding a synchronous or a metachronous approach, I think it is a matter of setting. Previously, when we did not have a hybrid operating room, we would have to cross half the hospital to come to the radiology suite to insert a stent-graft, because we had no ability to do it in the operating room. In our current setting, we do have this ability, and this is why we routinely perform it synchronously now.

Dr Coselli. I really hate to see political issues get in the way of the care of a patient. I know I am part of a dying breed, but the way we have solved it in my unit is that I am the vascular surgeon as well as the cardiac surgeon. So the decision is made at the time, and everything is taken care of under a single anesthetic.

Dr Miller. Dr Griepp, how is your blood pressure after hearing some of these presentations?

Dr Griepp. A lot of these things have great potential for the future. The present is not quite so wonderful, but we do have hopes that they will get better as time goes on.

Dr Abraham. Joe, can I just say one thing just to address Dr Miller's comments? He made a remark about my finding a cardiac surgeon to do these cases. I just want to make sure that everybody is clear: I don't go out trying to find cardiac surgeons to work on these patients; it is the cardiac surgeons who are contacting me. Mark Peterson in Toronto, Marc Pelletier from New Brunswick, Mike Moon in Winnipeg: I can tell you that there are not many cardiac surgeons better than those surgeons in those cities. So it might be difficult for me to find a cardiac surgeon, but perhaps I could send them to Stanford.

Dr Miller. Or you can send them to Baja Oklahoma. I am sure there is someone in Houston who would help out.

Dr Bavaria. I would back him up. Most of the cases that were presented in the Cook trial were actually referred by cardiac surgery.

Dr Jonathan Nwiloh (Atlanta, Ga). My question is for Dr Griepp. You had mentioned significant differences between hypothermic cardiac arrest (HCA) and non-HCA groups in terms of postoperative neurocognitive function. I wanted to know whether that difference normalizes with time, or whether it continues to persist into the long term?

A second question concerns your mentioning that the safe time for circulatory arrest is about 25 to 30 minutes, and I was wondering whether retrograde cerebral perfusion (RCP) or selective antegrade cerebral perfusion (ACP) extends that safety margin, and if so for how long?

Dr Griepp. Did your first question refer to cognitive testing?

Dr Nwiloh. Yes: whether the postoperative differences in cognitive testing persist with time or whether the difference between the 2 groups in your series normalizes.
Dr Griepp. Those figures that I showed you were from late tests. Some of those patients were tested within a week or so, but the quoted results were from patients 2 weeks postoperatively to as long as 6 months after surgery: we didn't have a further test at 1 year. We did see some change in those patients in whom we did early tests and late tests, but I think the quoted results probably are reasonably representative in terms of persistence.

One of the problems is that the further out you go, the more difficult it is to know what it means, because there are other changes that are going on. I suspect that if you took these same patients and tested them 2 years later, they would probably all do worse because they are older and many of them have atherosclerosis and so forth. So there is no perfect answer in terms of when to test, but we were convinced on the basis of this and on some subsequent studies that there are real decrements in performance. I would remind you that in routine cardiac surgical patients, and in patients with short periods of circulatory arrest, we didn't see any deterioration whatsoever.

Dr Bavaria. The second question was about RCP and HCA and whether RCP can increase that safe time as an adjunct to ACP.

Dr Griepp. Well, I can give you my prejudices that are based on some laboratory data and some very limited clinical experience. There is no evidence that RCP provides nutritive support to the capillaries of the brain in the experimental animal, and I think that is probably also true in human beings. I think that RCP can be helpful during periods of hypothermic circulatory arrest as an adjunct to cooling, but I think that we have shown in the experimental animal-and I suspect it is also true in human beingsthat you can keep the brain just as cold by packing the head in ice as with RCP. I'm sorry that Dr Safi isn't here. He is among the last holdouts of RCP to defend that practice. But I don't believe RCP does much good.

In terms of ACP, I think that is an entirely different situation. I think ACP is not HCA, and I suspect that you can go on for quite long intervals with ACP. Indeed, I think in most of the clinical series that I have seen, and from people that I have talked to who use a lot of ACP, the major issue having to do with duration has to do with distal body HCA. And if you are going to put a catheter in the descending aorta while you do ACP, you are basically doing cardiopulmonary bypass. I think if you want to use long periods of selective cerebral perfusion without distal perfusion, you have to be pretty cold. But on the basis of our experience with cold selective cerebral perfusion-and Dr Bachet can speak to this, although he never went as long as we did-you can probably go for an hour and a half, maybe as long as 2 hours, without any difficulty.

Dr Santi Trimarchi (San Donato, Italy). I have a question for Dr Yokoi. First, thank you very much for your presentation, which looks extraordinary. You report on about 400 patients. It looks very curious if we compare your results with the results from Dr Abraham, who used a kind of conventional branched graft. You reported about the fenestrated graft, but if I understood correctly, you don't have any side branch. I would like to know something more about what type of stent you have. Is it nitinol? Do you use balloon dilatation in these patients? What type of oversizing do you use? And what are your results in terms of endoleak and the long-term results? What is your longest follow-up? 
Dr Yokoi. I am not well experienced in this; I have never used a branched graft, because many surgeons have a special technique in Japan. We must limit embolic stroke and the complications within open surgical repair. So my investigation is very simplified, and safety is the most important issue. Stent-grafting is one of the therapies for thoracic aortic disease. Safety is the most important aim, and the second is the right indication. These patients are not fit for surgical therapy. During deployment, we use blood pressure to expand the stent-graft to the proximal landing zone, and $70 \%$ of patients don't need touch-up balloon dilatation.

Dr Bavaria. I think this is a multicenter national trial for Japan, I am sure it will be published pretty soon, and we will have to wait for that information for more detail.

Dr George Tolis, Jr (Boston, Mass). I have a question for Dr Spielvogel. In terms of the trifurcated graft technique, which you and Dr Griepp taught me how to do, I have noticed that it seems to be easier to just do the subclavian artery under circulatory arrest and then clamp the distal stumps of the left common carotid and the brachiocephalic artery and do these under blind selective perfusion through the axillary cannula at about $10 \mathrm{~mL} /$ $\mathrm{kg} / \mathrm{min}$. That decreases the circulatory arrest time a fair amount. You end up having about 8 or 10 minutes of circulatory arrest, the time that it takes to dissect out the arch and do the subclavian anastomosis, which is the hardest one to do. And then you convert the rest of the 20 minutes or whatever that would normally be circulatory arrest into blind unilateral selective perfusion. Do you think there is any problem with doing it this way? Do you think there are any drawbacks?

Dr Spielvogel. Actually, George, I think that your approach is right on the money, absolutely. I always think that the left subclavian is the most difficult to access when you are doing arch replacement; sometimes it's really displaced away from you. So actually that's exactly what I do; I always do the left subclavian first. And as time has gone on, we have gotten to the approach in which you begin perfusion unilaterally and just occlude the brachiocephalic vessels and sew the rest of the graft on. So, yes, you can do it in less than 10 minutes. I almost always do the left subclavian under circulatory arrest; it just seems easier.

Dr Tolis. You can inspect the vessels and see whether they are diseased. If they are diseased, then obviously you wouldn't clamp them, but usually the distal stumps are fairly disease free in the arches, at least in my experience.

Dr Spielvogel. Absolutely. I agree.

Dr Coselli. We do something quite similar. We use a trifurcation graft, and we will go to the left subclavian while we are cooling, and then you can perfuse through the trifurcation graft into the left subclavian, so that you are hitting your vertebral while you are doing the left carotid, and you are meanwhile perfusing through the axillary artery into the right carotid. And then under circulatory arrest you move the innominate artery, and while you are doing the innominate, you are perfusing all the head vessels. And so it adds body circulatory arrest time, but your cerebral circulatory arrest is over at that point. It really brings cerebral circulatory arrest time down to just a few minutes while you are doing the left carotid.

Dr Tolis. And under deep hypothermia or relative hypothermia?

Dr Coselli. We use $24^{\circ} \mathrm{C}$. We fall between some of those that are interested in perfusion at the higher levels of temperature, but we have a great deal of respect for some of the data that has come out of the group at Mount Sinai, so we have arbitrarily gone to about $24^{\circ} \mathrm{C}$.

Dr Tolis. So I would assume that under deep hypothermia you wouldn't be nervous about the circulatory arrest time involving the vertebral?

Dr Coselli. Certainly less so. I also think, however, that there is a group of patients in whom, during the cooling period, it is going to be hard to get to that left subclavian. So I think you need to look at the anatomic considerations, and there may be some people who should have carotid-subclavian bypass to eliminate that factor. Again, however, that is a patient selection issue.

Let me add just one more thing to that. I haven't heard it mentioned yet, but we have run into some patients with large vertebral arteries coming off the transverse aortic arch and have elected to maintain patency of those. On occasion, we have reattached them to that Dacron polyester fabric graft that goes off to the left subclavian, but more often than not we just reattach them to the left carotid.

Dr Eric Roselli (Cleveland, Ohio). First, I would like to make a comment with regard to the discussion that went on earlier about antegrade delivery of stent-grafts. In our experience, we have had a couple of patients who had some spinal cord ischemia related to extensive coverage with the antegrade delivery of stent-grafts. And so we have learned, despite having cerebrospinal fluid drainage, that if we have to cover an extensive amount of thoracic aorta, it is probably best, as Dr Griepp has taught us, to break up that insult over time and do that second operation retrograde maybe 5 days or a week later.

I also have a question for Dr Yokoi about the fenestrating device. Those are fascinating images, but what sort of rescue strategies do you have in place in the event that you can't get access or can't get the fenestrations lined up with those vessels? It didn't look like there were very many wires in those aortas on those images you showed.

Dr Yokoi. We have 3 chimney stents in the operation, and the alignment - the rotation of the device-is no problem because we have a lot of experience. The problem is if it is too proximal or too distal. If too proximal, then after deployment we can put a stent from the left common carotid into the sheath with bare stent chimney stenting. We have no complications, or almost no complications, because we have a lot of cases.

Dr Roselli. So in the 3 patients that you had to salvage, you were able to access the fenestrations with a stent?

D. Yokoi. Yes.

Dr Roselli. Are the fenestrations on the device custom designed for the patients?

Dr Yokoi. They are custom designed, but we have already settings prepared, so that the stent can be fabricated very quickly just by selecting from the various types of fenestrations. So we can start a clinical trial.

Dr Roselli. So there are fewer variations in the arch than in the thoracoabdominal aortic branches?

Dr Yokoi. Yes.

Dr Jehangir Appoo (Calgary, Alberta, Canada). I have 2 questions, 1 for Dr Yokoi and 1 for Dr Hagl. Dr Yokoi, I am very impressed that you have this series of 350 totally endovascular arches. It is very impressive. I noticed I think that 3 of them 
were for aortic dissection. I was wondering if these were for acute aortic dissections and whether you are moving toward using this therapy in the acute situation.

I also wanted to ask Dr Hagl what their standard approach is for an acute type A dissection and their indications for an elephant trunk in acute type A dissection.

Dr Yokoi. Three patients had previously had type A chronic aortic dissection. They just had little endoleaks after the procedure, so we were required to perform touch-up ballooning. One patient is currently in conservative follow-up, and 2 patients were taken to open surgery.

Dr Appoo. Would you consider using this for an acute type A dissection?

Dr Yokoi. I think that is the next program for me.

Dr Hagl. I think the most important thing in a type A aortic dissection is that the patient survives. That is the first goal that you have to achieve. So you need some experience with the frozen elephant trunk, for example, to consider using it in a type A aortic dissection. But we are starting to get more and more aggressive in using it, especially if there are tears in the aortic arch. If there are no arch tears when you open the arch, we go with a proximal arch repair. If we see tears in the distal arch, we do a frozen elephant trunk. I have to confess, though, that we don't know what is perfect sizing, and we are still trying to figure that out.

Dr Bavaria. I have a couple of questions as well. This is for our Japanese surgeons, as well as Dr Griepp maybe. This issue was raised by Dr Coselli. We have a couple of groups, maybe one major group, that uses circulatory arrest alone for all arch cases, a number of groups that use RCP alone. Are RCP and straight HCA dead at this point? I noticed in the Japanese literature, Dr Okita, that $10 \%$ or $15 \%$ of the surgeons still use RCP. Can you comment on that? Then maybe we can go down the panel with the arch surgeons. Is it all ACP now, with everything else dead? Or not?

Dr Okita. I only use deep HCA and RCP on very simple cases and hemiarches.

Dr Bavaria. This is important. So you use RCP for straightforward open distal anastomoses, hemiarches, and ACP for all total arches?

Dr Okita. In selected patients. For complicated cases or a very sick patient, I always use ACP in hemiarch repair, because we switched to ACP from RCP. We had a circulatory arrest time of 40 or 50 minutes with RCP with HCA; we had a lot of patients with transient neurologic deficits. So we changed to ACP.

Dr Bavaria. So that is Dr Kazui's paradigm, too: if circulatory arrest time is less than 30 minutes for a hemiarch then use RCP; for everything else, ACP.

Dr Okita. No, shorter than that: 20 minutes.

Dr Ogino. Our approach is different. I use ACP for all cases. Our technique is very simple: right axillary artery cannulation and femoral cannulation. This technique we use for all patients, even with aortic dissection. We call this double cannulation. It is very simple. It is quite easy; just a clamp on the innominate artery as ACP is started. So our basic method is ACP.

Dr Bavaria. So, Dr Coselli, do you do ACP even for 20-minute circulatory arrest times?

Dr Coselli. We quit using RCP years ago, just abandoned it. Since we moved to axillary and innominate artery sites for cannulation, not to use ACP in some form or fashion, even for a short period of circulatory arrest, is not very appealing.

Dr Bavaria. Roberto, and this is also for the panel if anybody wants to join in, regarding reoperations and the whole concept of left ventricular venting. It seems that many surgeons kind of blow that off. They don't look at it as a very important concept, whereas many of us actually feel it is really critical, even to the point, as you showed, of doing a small left thoracotomy. Can you tell us about your approaches to left ventricular venting in the reoperations? What are your thoughts? Sometimes it is hard to get to anything.

Dr Di Bartolomeo. When reentry into the sternum is difficult, and if there is severe aortic valve insufficiency, I think it is very important to put the vent in the left ventricle. It is a very simple technique to introduce a small vent in the apex of the left ventricle.

Another important thing about which I think I spoke during the presentation is the isolation of the innominate artery before opening the sternum. Because in these cases when you arrive at the temperature you want $-26^{\circ} \mathrm{C}, 25^{\circ} \mathrm{C}, 24^{\circ} \mathrm{C}$-you put a clamp on the innominate artery and reduce cardiopulmonary bypass flow; generally, I use $800 \mathrm{~mL}$ in the left axillary artery. In these cases, you have perfusion of the cerebral regions and in the majority of the cases also the left hemisphere and then can open the sternum.

Dr Bavaria. Dr Griepp? Again, it is very difficult sometimes in these really bad reoperations to get into venting anatomies.

Dr Griepp. I actually wanted to comment on something else. I want to say a word about the issue of paraplegia and elephant trunks and frozen elephant trunks. It started out being a tremendous conundrum to me that in doing descending thoracic aortic open surgery, one only extremely rarely saw a spinal cord insult from a resection limited to the chest. I don't buy the concept that it is the elephant trunk occluding segmental vessels that's causing the paraplegia.

There are basically three issues that I think need to be kept in mind if this $5 \%$ or $7 \%$ or sometimes $3 \%$ incidence of paraplegia in elephant trunks is going to be dealt with.

One concerns perfusion of the left subclavian: the problem of leaving the left subclavian without perfusion for the long term. There is lots of experimental evidence and some from clinical series too, that you need the left subclavian if you are going to take out a number of segmental vessels. So that's the first reason one might get paraplegia with an elephant trunk. The second is the injury to the spinal cord from lower body circulatory arrest at warmer temperatures. In many cases, there is a sublethal injury delivered to the cord. It may be only 30 minutes at $30^{\circ} \mathrm{C}$ or something like that. The cord may recover under optimal circumstances, but then on top of that you have a patient who has undergone a big, complex operation and who is probably not going to be hemodynamically stable to the same extent that your descending thoracic open case has been. The patient may be intubated for a couple of days, and nobody is going to be paying attention to keeping the blood pressure up. Particularly if there is a little bleeding, the blood pressure is allowed to drift down. So then you take the combination of a sublethal injury from not perfusing the cord, or particularly the lower part of the cord, during selective cerebral perfusion and then on top of that you superimpose some instability in postoperative hemodynamics. I think those are probably the main considerations in causing spinal cord injury in these patients. I would hope that 
attention to those particular issues might reduce the incidence of spinal cord injury with elephant trunks.

Dr Miller. I just would like to give an answer to your point about reoperations. Roberto, I agree $100 \%$. You have the chronic third- and fourth-time reoperations with a lot of aortic regurgitation. You have to open the sternum under circulatory arrest. You can make the little thoracotomy you described, but for 5 or 10 years we have just been venting the apex percutaneously with transesophageal echocardiographic guidance. Remember, those of you who were at the National Institutes of Health in the old days; all could do a percutaneous left ventricular stick. The points of maximal impulse are really out there, the ventricle is big, and over a wire one can just put in an 18F EOPA cannula or something like that, and then later on dissect it out and put a stitch in it. This has been part of the TEVAR experience, as I think you have shown us. Someday we are going to be doing percutaneous transapical TEVARs.

Dr Bavaria. Do you mean transcatheter aortic valve implantations?

Dr Miller. Transcatheter aortic valve implantations. But you have done a TEVAR through the apex too. More than one?

Dr Bavaria. More than one. 